Perspectivas sobre la enseñanza de la gramática: Currículum, propuestas didácticas y práctica de aula

\title{
ACTIVIDAD METALINGÜÍSTICA Y REFLEXIÓN DURANTE LA ESCRITURA COOPERATIVA EN SECUNDARIA
}

\author{
Recepción: 01/04/2020 | Revisión: 01/05/2020 | Aceptación: 02/09/2020
}

\begin{abstract}
Aina REIG
Universitat de València

Aina.Reig@uv.es actividad metalingüística y la reflexión sobre la lengua, el texto y el discurso.

\section{METALINGUISTIC ACTIVITY AND REFLECTION DURING COOPERATIVE WRITING IN SECONDARY} EDUCATION

Abstract: This article presents the main results of a study that analyses the metalinguistic activity during the writing composition process in small groups and within the framework of an instructional sequence (IS). The sequence aims at the elaboration of an academic text from different sources of documentation. Data is, therefore, extracted from the conversations of two work teams during cooperative writing over three sessions. Between the first two sessions and the third one, external regulation dynamics are introduced. The research shows the moments in which the metalinguistic activity occurs, it allows to know the content of this activity as well as its evolution. It also points out some factors that promote the metalinguistic activity and the reflection on language, text and discourse.
\end{abstract}

Resumen: Este artículo presenta los principales resultados de un estudio que se propone el análisis de la actividad metalingüística durante el proceso de composición escrita en pequeño grupo y en el marco de una secuencia didáctica. La secuencia tiene como objetivo la elaboración de un texto académico a partir de la lectura de diferentes fuentes de documentación. Los datos se extraen, por lo tanto, de las conversaciones de dos equipos durante la escritura cooperativa a lo largo de tres sesiones. Entre las dos primeras y la tercera se introducen dinámicas de regulación externa. El trabajo muestra en qué momentos de la SD se produce la actividad metalingüística, permite conocer el contenido de esta actividad y su evolución, y señala algunos de los factores que fomentan la

Palabras clave: actividad metalingüística; composición escrita; secuencias didácticas; interacción; regulación metacognitiva.

Keywords: metalinguistic activity; writing composition; instructional sequences; interaction; metacognitive regulation.

\section{ACTIVITAT METALINGÜÍSTICA I REFLEXIO DURANT L'ESCRIPTURA COOPERATIVA A SECUNDARIA}

Resum: Aquest article presenta els resultats principals d'un estudi que es proposa lanàlisi de lactivitat metalingüística durant el procés de composició escrita en petit grup i en el marc d’una seqüència didàctica. La seqüència té com a objectiu l'elaboració d'un text acadèmic a partir de la lectura de diferents fonts de documentació. Les dades s'extrauen, per tant, de les converses de dos equips durant lescriptura cooperativa durant tres sessions. Entre les dues primeres i la tercera s'introdueixen dinàmiques de regulació externa. El treball mostra en quins moments de la SD es produeix l'activitat metalingüística, permet conèixer el contingut d'aquesta activitat i la seva evolució, $i$ assenyala alguns dels factors que fomenten l'activitat metalingüistica i la reflexió sobre la llengua, el text i el discurs.

Paraules clau: activitat metalingüística; composició escrita; seqüències didàctiques; interacció; regulació metacognitiva. 


\section{Introducción}

Saber lengua implica ser capaz de interactuar de manera efectiva en las diferentes esferas de la vida social, lo que requiere ejecutar las diferentes habilidades necesarias para comunicarse (Camps, 2020; Zayas, 2012a). Sin embargo, para aprender lengua no basta con usarla. Los usos escritos de la lengua y los usos orales formales requieren un conocimiento explícito del sistema lingüístico. Para su aprendizaje es necesario activar un alto grado de consciencia y convertir la lengua en objeto de reflexión (Camps, 2017; Fontich, 2016; Rodríguez Gonzalo, 2012). En este sentido, la actividad de composición escrita constituye un escenario idóneo para el aprendizaje de la lengua y de sus usos (Camps y Milian, 2020; Fontich y Camps, 2014; Myhill, 2011).

Con este trabajo ${ }^{1}$ pretendemos mostrar las conexiones entre la actividad metalingüística y la composición escrita. La implementación de un proyecto de escritura basado en el modelo de secuencias didácticas (Camps, 2003, 2020) enmarca el desarrollo de actividades de lectura y escritura orientadas a la elaboración en pequeño grupo de un texto académico. En este artículo presentamos el análisis de la conversación de dos equipos durante la escritura cooperativa de dicho texto, es decir, mientras hablan sobre la tarea, sobre la escritura y sobre lo escrito. Todo ello se efectúa desde la perspectiva del proceso de aprendizaje, teniendo en cuenta la evolución del trabajo a lo largo de tres sesiones. A pesar de que no se prevé una enseñanza explícita de la gramática, la secuencia incluye dinámicas de revisión entre iguales y de reescritura centradas en aspectos lingüísticos, textuales y discursivos concretos, siempre vinculados al texto que se está produciendo y a la tarea prevista.

Los objetivos que nos planteamos son: 1) Detectar en qué momentos de la secuencia didáctica se produce la actividad metalingüística. 2) Conocer el contenido de esta actividad y su evolución a lo largo de la secuencia. 3) Identificar los factores que fomentan la actividad metalingüística.

\section{Marco teórico}

Numerosos estudios han subrayado la relación entre la composición escrita y la actividad metalingüística (Camps y Milian, 2020; Fontich y Camps, 2014; Myhill, 2011; Ribas, 2020). Escribir consiste en operar sobre las formas lingüísticas y conlleva una toma de decisiones constante, por eso precisa reflexionar sobre la lengua y sobre lo que se escribe. Estas decisiones afectan a niveles lingüísticos diversos, y pueden ser metaléxicas, metasemánticas, metatextuales y metapragmáticas (Gombert, 1990).

La actividad metalingüística recoge diferentes acepciones. Autores como Culioli (1990) diferencian las representaciones lingüísticas de las metalingüísticas y sitúan las metalingüísticas en el nivel explícito. Desde esta perspectiva, la actividad metalingüística es siempre consciente, a diferencia de la que se produce involuntariamente, bautizada como epilingüística. En cambio, para autores como Karmiloff-Smith (1992), la actividad metalingüística puede ser consciente e

1 Este trabajo se ha desarrollado en el marco del Programa Nacional de Formación del Profesorado Universitario del Ministerio de Ciencia, Innovación y Universidades del Gobierno de España (convocatoria FPU2015). 
inconsciente. Nosotros nos situamos en la línea de los trabajos del grupo GREAL, ${ }^{2}$ para el que la actividad metalingüística es toda manifestación visible de la actividad mental, verbal o no verbal, relacionada con la lengua y su uso (Camps, 2017; Camps y Milian, 2020; Fontich y Camps, 2014). Para el GREAL, la actividad metalingüística se manifiesta en tres niveles diferentes de explicitud y puede ser procedimental o declarativa, verbal o no verbal (Camps et al., 2020). Cuando es no verbal (primer nivel), se observa a través del proceso de reformulación, de repeticiones y de énfasis. En cambio, si se verbaliza, puede hacerse con un lenguaje común (segundo nivel) o con términos lingüísticos específicos (tercer nivel). Pero estos términos lingüísticos no se refieren únicamente a la gramática. El desarrollo metalingüístico durante la escritura requiere, además de los saberes gramaticales, saberes socioculturales y metacognitivos, es decir, sobre el género y sobre el proceso (Myhill y Jones, 2015). Asimismo, el metalenguaje usado durante la escritura puede ser gramatical, literario, referido al género o sobre el proceso (Myhill, 2011).

Mediante la escritura, la lengua se materializa, lo que permite observarla, explorarla a través de los usos y construir conocimiento. Desde la perspectiva del aula, la investigación ha demostrado que el proceso de escritura colaborativa promueve la actividad metalingüística (Camps et al., 2020; Fontich y García Folgado, 2018). El modelo de secuencia didáctica de Camps (2003, 2020) plantea un proyecto de trabajo con unos objetivos y unos contenidos discursivos y de aprendizaje explícitos y vinculados a la producción de un género discursivo. Esta propuesta reposa sobre el trabajo cooperativo, la evaluación formativa y las tareas comunicativas. En primer lugar, la escritura en grupo obliga a compartir el proceso y los objetivos. Fuerza, por consiguiente, a verbalizar los procesos y a explicitar las reflexiones sobre la forma, el contenido y el contexto para poder tomar decisiones que permitan la consecución de la tarea. El diálogo entre iguales puede hacer más consciente el proceso y, por lo tanto, puede promover su control (Mercer y Littleton, 2007). En segundo lugar, y vinculada a la idea del control, encontramos la evaluación formativa, mediante la que se pretende que el aprendiz tome conciencia de los procesos de aprendizaje y sea capaz de autorregularlos. El intercambio verbal con los demás y los instrumentos de regulación externa, como parte de la evaluación formativa, pueden promover la reflexión sobre lo que se aprende y la regulación metacognitiva (Allal, 2000; Ribas, 2000, 2020). En tercer lugar, la orientación comunicativa de las tareas de escritura pone en conexión lengua y uso, ya que lo que da sentido a la producción es la situación de comunicación real. Los propósitos comunicativos asociados a unos ámbitos de uso llevan aparejados unos determinados géneros discursivos. La interacción con los géneros exige conocer los rasgos lingüísticos y textuales que los caracterizan (Zayas, 2012a). Cuando la lectura y la escritura son situadas, los conocimientos sobre lengua se ponen al servicio de un acto comunicativo particular.

Propuestas como la de Camps (2003) conciben la lectura y la escritura como dos actividades interrelacionadas que no solo permiten acceder a la información y transmitirla, sino también elaborar y transformar el conocimiento (Bereiter y Scardamalia, 1987). Los escolares, a lo largo de su formación, viven situaciones de enseñanza y aprendizaje que los preparan para ingresar en la cultura escrita. Uno de los ámbitos de uso al que acceden es el académico, para el que tienen que

2 El grupo GREAL (Grup de Recerca sobre l'Ensenyament i Aprenentatge de Llengües) de la Universitat Autònoma de Barcelona. 
desarrollar maneras específicas de leer, escribir y usar el lenguaje (Carlino, 2013). La alfabetización académica requiere el tratamiento integrado de las prácticas de lectura y escritura. En el aula, esto se materializa en las llamadas tareas híbridas o tareas de leer para escribir y escribir basándose en la lectura de fuentes. La enseñanza integrada de ambos procesos potencia el dominio de las habilidades lectoescritoras y promueve el desarrollo cognitivo (Flower, 1990; Graham et al., 2018). Por otro lado, alfabetizarse académicamente implica apropiarse de las convenciones y usos característicos de los géneros propios de este ámbito. En consecuencia, la actividad discursiva efectuada en la interacción con estos textos exige conocer las características de los géneros y saber reflexionar sobre el contenido y sobre las formas lingüísticas y textuales que los singularizan (Zayas, 2012b).

\section{Metodología}

Esta investigación es un estudio de caso basado en la observación participante en el que se implican dos grupos-clase de $4^{\circ}$ de ESO (15-16 años) con sus respectivos profesores de lengua catalana. $\mathrm{El}$ análisis consiste principalmente en un tratamiento cualitativo de los datos que se combina con un tratamiento cuantitativo. Los datos se recogen de una intervención de aula en forma de proyecto de trabajo inspirado en la propuesta de secuencias didácticas (SD) para aprender a escribir de Camps $(2003,2020)$.

\subsection{La secuencia didáctica}

La secuencia didáctica, de seis sesiones, consiste en la escritura cooperativa de un texto académico de temática histórica que se nutre de la información hallada en diferentes textos en un dossier de fuentes preparado para esta ocasión. Siguiendo a Camps (2020), las prácticas de lectura y de escritura se enmarcan en una situación comunicativa que da sentido al texto que tienen que producir: los alumnos deben informarse a partir de estas fuentes para escribir un texto propio y original que sirva para informar a los estudiantes de otro centro sobre el tema histórico en cuestión. Estos destinatarios reales no dispondrán del dossier con el que se tienen que documentar nuestros alumnos; el texto a producir, pues, deberá ser autónomo.

La SD se inicia con la presentación del proyecto y la representación de la tarea, y concluye con la escritura del texto definitivo. Se divide en diferentes sesiones, entre las que se suceden dinámicas de lectura y escritura: análisis en gran grupo de un texto modelo y lectura de textos introductorios en gran grupo (sesión 1), lectura inicial del dossier en pequeño grupo (sesión 2), escritura del primer borrador y de textos intermedios (sesiones 3 y 4) y dinámicas de revisión entre iguales (sesión 5) para preparar la escritura final (sesión 6). Como se observa, para reforzar las etapas del proceso de lectura y escritura y facilitar su control por parte de los estudiantes se introducen los siguientes mecanismos de regulación externa:

- Análisis de un texto modelo y guion de escritura (sesión 1). Tras el análisis en gran grupo del texto modelo se consensuan unas bases para la composición del texto propio, a partir de las que se crea un guion de apoyo a la escritura. 
- Dinámicas de revisión entre iguales y ficha de revisión (sesión 5). Cuando concluye la escritura del borrador o primera versión, cada grupo revisa el texto de otro equipo y rellena la ficha de revisión. Después, ambos equipos exponen la revisión y ponen en común las observaciones.

- Consejos de escritura de la versión final (sesión 6). Tras la sesión de revisión entre iguales se les entrega un documento que recoge orientaciones personalizadas de reescritura.

Todos los instrumentos de regulación incluyen observaciones sobre la gramática del texto, el contenido, la estructura y la expresión.

\subsection{Enunciados con función metalingüística (EM)}

Los resultados que exponemos en este artículo proceden del análisis de la interacción oral de dos grupos, que hemos llamado G2 y G5, durante las tres sesiones en las que se dedican exclusivamente a la redacción de los textos: las sesiones tercera, cuarta y sexta (a partir de ahora, S3, S4 y S6, respectivamente). Concretamente, nos fijamos en las intervenciones que tienen que ver con la tarea, es decir, con aspectos textuales, lingüísticos y discursivos. Una vez identificados dichos enunciados, los clasificamos siguiendo la propuesta de Camps et al. (2020) y Ribas (2020) para la categorización de enunciados metalingüísticos (EM), haciendo una adaptación para el tipo de tarea de aula planteada. Como en nuestra SD el contenido temático es fundamental para la construcción del texto, tenemos en cuenta los comentarios de este tipo siempre que se vinculen a alguna cuestión relacionada con el contexto discursivo, el texto (la superestructura, el género, las partes del texto, la generalización y la ejemplificación, etc.) o la lengua (gramática, ortografía, etc.).

- Los aspectos discursivos (AD). Engloba aquellos comentarios que se refieren al contexto de producción y de recepción: escritor / enunciador, lector / destinatario, intención, registro, modalización, léxico marcado, etc.

- La superestructura (SE). Incluye las intervenciones que tienen que ver con la organización y la superestructura del texto que se ha de producir: esquema textual, género, título, introducción, desarrollo y conclusión.

- El contenido semántico (CS). Abarca los comentarios que remiten a la macroestructura en cuanto a la coherencia interna, la coherencia respecto a un referente, la precisión del significado, la valoración de un contenido y la traducción de un término (catalán / español).

- La gramática del texto y de la frase (G). Comprende los enunciados en los que se tratan cuestiones como la construcción sintáctica, la flexión, la conexión, la puntuación y la segmentación del texto, y otros mecanismos cohesivos.

- Los aspectos gráficos y sonoros (AGS). Engloba las intervenciones sobre la ortografía, la forma sonora, los aspectos tipográficos y la extensión.

- El texto en su globalidad y las valoraciones (GV). Incluye las valoraciones del texto en su globalidad o de aspectos particulares.

En la descripción y análisis de las secuencias con contenido metalingüístico, consideramos también las reformulaciones al hilo de la conversación, es decir, mientras los alumnos hacen 
propuestas de escritura o de revisión del texto. Según Camps et al. (2020) y Camps y Milian (2020), las reformulaciones pueden ser: simples (propuesta de cambio sin justificación), con negación explícita (se rechaza la propuesta de un compañero y se hace una nueva propuesta), con reflexión pero sin metalenguaje y con metalenguaje.

El G2 lo forman tres estudiantes de rendimiento académico medio-bajo (Marina y Teo) y bajo (Rosa), y el G5 lo componen Víctor y Juan Luis, una pareja de nivel bajo. Ambos grupos comparten la dificultad inicial en la representación mental de la tarea y la motivación por el trabajo, que aumenta sesión tras sesión. Además, los grupos siguen ritmos de trabajo distintos y obtienen resultados dispares. Una diferencia fundamental entre ambos es la colaboración y el grado de implicación. En el G2 se produce una evolución evidente: en la S3 no trabajan cooperativamente, en la S4 Marina se responsabiliza de la escritura y dicta una nueva versión del texto a su compañera mientras Teo introduce constantes digresiones, y en la S6 Marina y Rosa se vuelcan enteramente en la redacción del texto final. Por otra parte, en el G5, Víctor y Juan Luis tienen un estilo colaborador pero su hábito de trabajo pobre provoca distracciones muy frecuentes y una desorientación generalizada.

\section{Resultados}

En este apartado presentamos los resultados de la investigación. Primeramente, mostramos los datos derivados del recuento de enunciados metalingüísticos (EM) de los dos equipos. Después, desarrollamos el análisis de las interacciones en dos bloques: primero, las de las dos primeras sesiones de escritura, y después las de la sesión final, momento en el que podrían tener efecto las principales regulaciones externas previstas por la SD.

\subsection{Recuento de enunciados metalingüísticos (EM)}

El cómputo, en números absolutos, de los EM de los dos grupos analizados (G2 y G5) durante las tres sesiones dedicadas íntegramente a la redacción muestra un comportamiento desigual entre el G2 y el G5 (ver Figura 1). Mientras que en el G2 las intervenciones con función metalingüística aumentan a medida que avanzan las sesiones de escritura, el G5 experimenta un descenso en la S4 y un aumento mayor en la sesión final. En ambos casos, en la sesión final se registra el número más elevado de EM. 


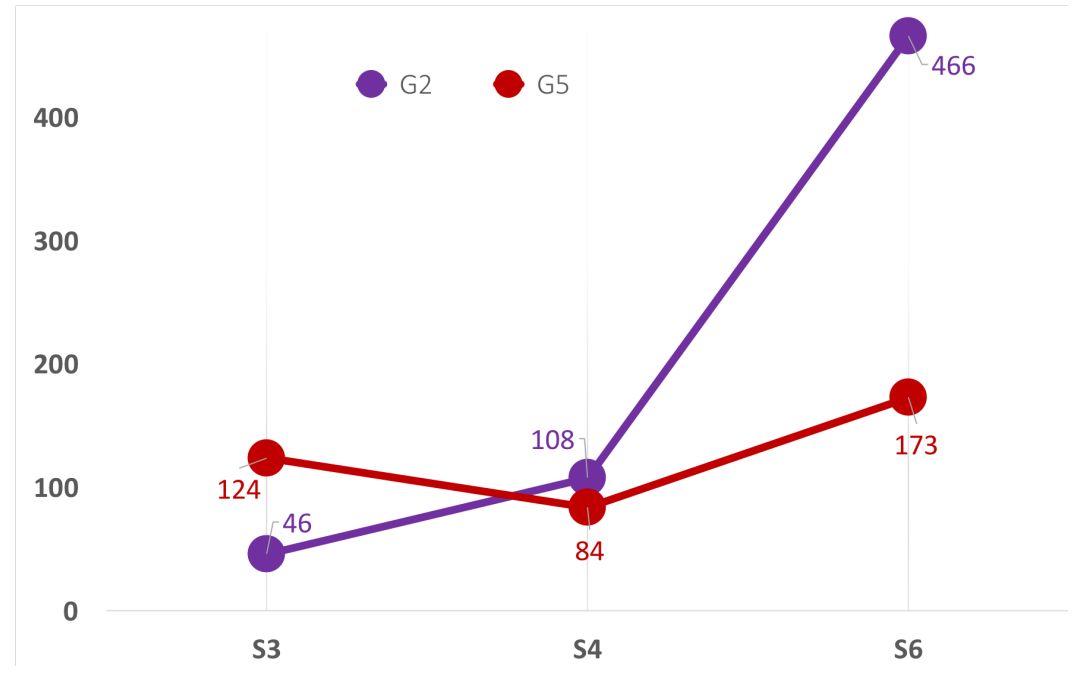

Figura 1. Comparativa de los enunciados metalingüísticos (EM) del G2 y del G5.

Basándonos en la atribución de las categorías descritas en el apartado de la metodología, realizamos el recuento de las intervenciones que contienen comentarios de cada tipo de EM (ver Figuras 2, 3 y 4). La Figura 2 muestra la distribución global de EM de los dos grupos por categorías. Como se observa, en ambos grupos se imponen las categorías de superestructura, contenido semántico y gramática, y son escasos o irrelevantes los comentarios sobre aspectos discursivos y las valoraciones de tipo general. Los resultados divergen en cuanto a los enunciados sobre aspectos gráficos y sonoros, puesto que para el G2 son los que registran un número mayor, mientras que para el G5 son escasos.

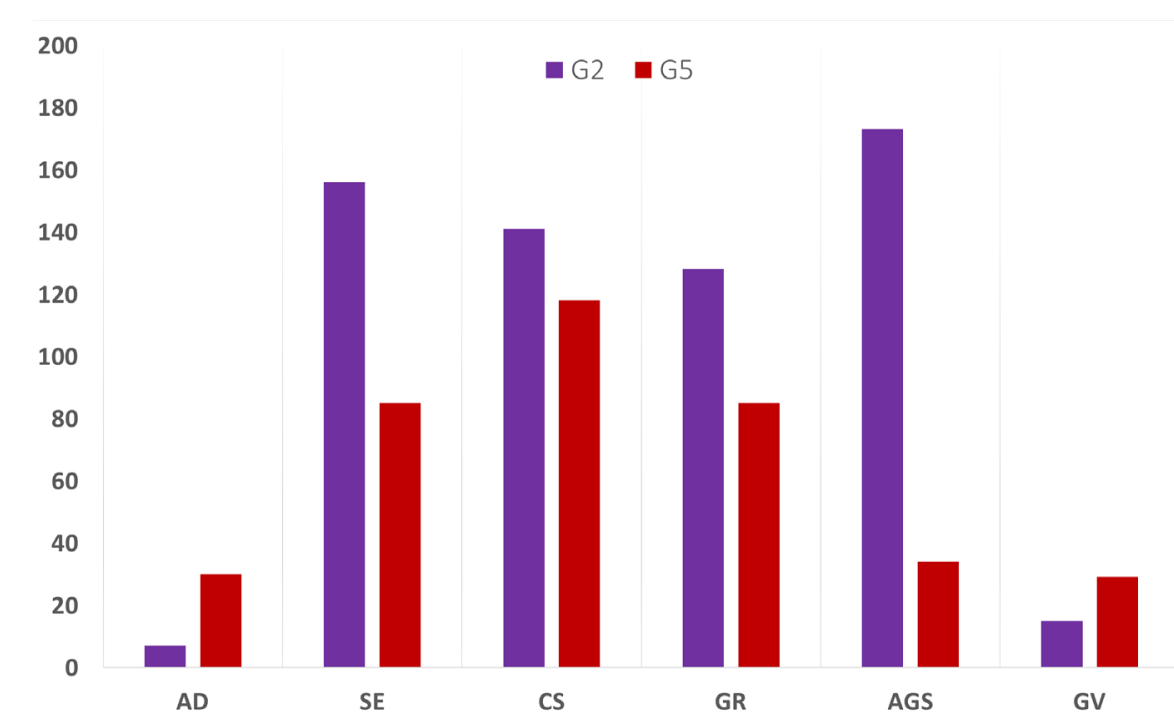

Figura 2. Comparativa de la tipología de enunciados metalingüísticos del G2 y del G5. ${ }^{3}$

3 Aspectos discursivos (AD), Superestructura (SE), Contenido semántico (CS), Gramática (GR), Aspectos gráficos y sonoros (AGS), Texto global y valoraciones (GV). 
Las Figuras 3 y 4 reflejan, respectivamente, la distribución de los valores absolutos de los EM por sesiones del G2 y del G5. Incluyen también los valores relativos de EM con respecto a los enunciados totales de cada sesión. La distribución de las cifras por sesiones permite detectar, en primer lugar, que se registran datos de algunas categorías en todas las sesiones mientras que hay otras que aparecen solamente en alguna o algunas de las sesiones de escritura. De igual modo, podemos afirmar que hay categorías poco representadas en un momento determinado de la secuencia didáctica y que, sin embargo, en otras sesiones tienen mucho más peso. Esto ocurre visiblemente en la S6, en la que todas las categorías están representadas y, además, con valores superiores u ostensiblemente superiores a las sesiones anteriores.

\begin{tabular}{|c|c|c|c|c|c|c|c|c|}
\hline & & $\$ 3$ & & & & & \multicolumn{2}{|c|}{ TOTAL } \\
\hline AD - Aspectos discursivos & & - & & & & & \multicolumn{2}{|c|}{7} \\
\hline SE - Superestructura & & 26 & & & & & \multicolumn{2}{|c|}{156} \\
\hline CS - Contenido semántico & & 5 & & & & & \multicolumn{2}{|c|}{141} \\
\hline GR - Gramática & & - & & & & & \multicolumn{2}{|c|}{128} \\
\hline AGS - Aspectos gráfic. y son. & & 12 & & & & & \multicolumn{2}{|c|}{173} \\
\hline GV - Texto global y valoración & & 3 & & & & & \multicolumn{2}{|c|}{15} \\
\hline Total & 46 & $30 \%$ & 108 & $25 \%$ & 466 & $74 \%$ & 620 & $51 \%$ \\
\hline
\end{tabular}

Figura 3. Enunciados metalingüísticos del G2.

Observamos también que, en términos relativos, la sesión en la que los EM aparecen con mayor frecuencia en las conversaciones de los alumnos del G2 es la sesión final (S6). En esta sesión, el contenido del 74\% de las intervenciones del grupo es metalingüístico. No podemos hacer extensible esta afirmación al G5. Si bien es cierto que, en lo referente al G5, en números absolutos, el número de EM de la S6 es superior al de las dos sesiones precedentes, en números relativos la diferencia entre la sesión inicial de redacción (S3) y la final (S6) es mínima.

\begin{tabular}{|c|c|c|c|c|c|c|c|c|}
\hline & & & & $\$ 4$ & & & \multicolumn{2}{|c|}{ TOTAL } \\
\hline AD - Aspectos discursivos & & & & - & & & \multicolumn{2}{|c|}{30} \\
\hline SE - Superestructura & & & & 16 & & & \multicolumn{2}{|c|}{85} \\
\hline CS - Contenido semántico & & & & 44 & & & \multicolumn{2}{|c|}{118} \\
\hline GR - Gramática & & & & 19 & & & \multicolumn{2}{|c|}{85} \\
\hline AGS - Aspectos gráfic. y son. & & & & 3 & & & \multicolumn{2}{|c|}{34} \\
\hline GV - Texto global y valoración & \multicolumn{2}{|c|}{8} & \multicolumn{2}{|c|}{2} & \multicolumn{2}{|c|}{19} & \multicolumn{2}{|c|}{29} \\
\hline Total & 124 & $28 \%$ & 84 & $19 \%$ & 173 & $32 \%$ & 381 & $27 \%$ \\
\hline
\end{tabular}

Figura 4. Enunciados metalingüísticos del G5. 


\subsection{Análisis de las interacciones durante las dos primeras sesiones de escritura}

La tercera sesión de la SD (S3) es la primera que se dedica a la redacción. A pesar de que los docentes han recapitulado las indicaciones sobre la tarea de escribir un texto a partir de las fuentes de documentación del dossier, tarea presentada al inicio de la SD, los grupos necesitan tiempo para situarse. El trabajo es de una innegable complejidad, puesto que los aprendices se enfrentan al doble reto de comprender los textos y elaborar un texto propio que se nutra de las lecturas que se les han facilitado, teniendo en cuenta el planteamiento comunicativo que da sentido a la tarea.

En la conversación de la S3 del G2, los enunciados sobre aspectos metalingüísticos son anecdóticos y se producen cuando un estudiante consulta a sus compañeros una duda ortográfica o cuando ponen en común el trabajo, ya que la colaboración es casi insignificante. Tal y como se observa en el fragmento de la Figura 5, en la formulación de la pregunta no se emplea el término apropiado (ce grande en vez de mayúscula), pese a que se trata de una noción general de uso habitual en el aula desde la educación primaria. Los otros dos miembros del grupo, Rosa y Teo, que tampoco recurren al término en cuestión, no muestran interés por dar solución al problema.

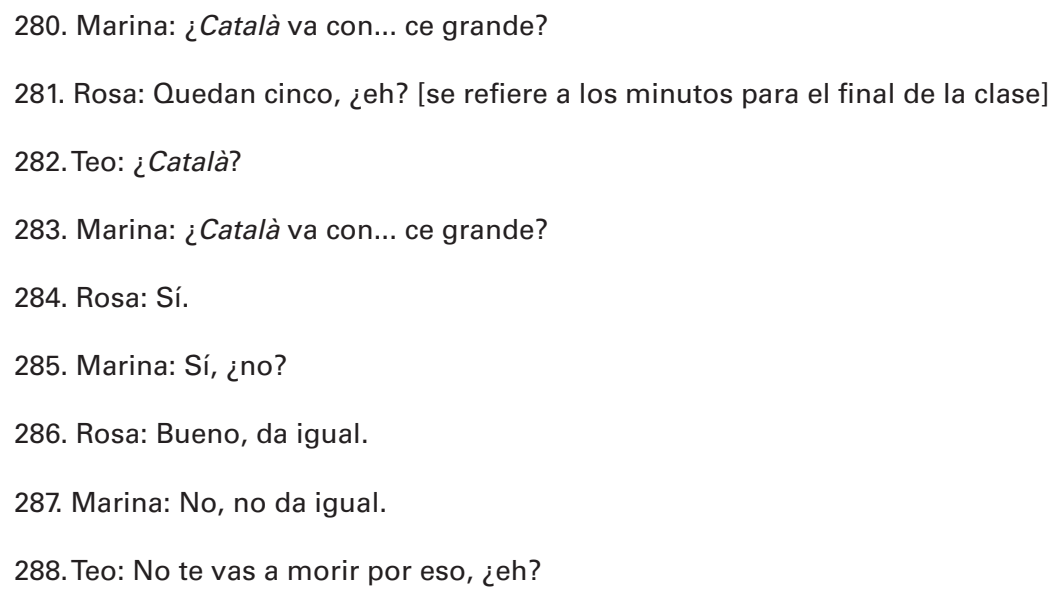

En la segunda sesión de redacción (S4), el G2 no se siente satisfecho con el trabajo del día anterior y decide escribir una segunda versión del texto. Esta vez, en vez de trabajar individualmente comparten la textualización. El cambio a la modalidad de trabajo colaborativa explica el aumento de los EM. Sin embargo, como la gestión del texto queda fundamentalmente en manos de Marina, no es fácil encontrar muestras de reflexión metalingüística explícita. Los ejemplos de esta sesión son similares a los de la sesión anterior. En el siguiente ejemplo (Figura 6), Marina, la alumna que asume la textualización, dicta el texto a su compañera Rosa. Nuevamente, se repiten dos elementos que caracterizan la conversación del grupo: no se emplean términos metalingüísticos apropiados y no se producen razonamientos sobre la organización del contenido del texto ni sobre la puntuación de la oración, ni para justificar decisiones (el punto y seguido que propone 
Marina) ni para argumentar alternativas (la coma que sugiere Teo). Los diferentes atributos para referirse al punto y seguido son no separado y en el mismo, en contraposición al punto y aparte, que nombran con el adverbio abajo. Ni siquiera la apelación al término adecuado por parte de Rosa (enunciado 406) provoca un cambio en los usos de su compañera.

400. Marina: Punto.

401. Teo: Coma.

402. Marina: Pero no separado.

403. Rosa: ¿Abajo?

404. Marina: No.

405. Teo: Ha dicho no separado.

406. Rosa: Punto y seguido.

$[\ldots]$

432. Marina: En el mismo.

433. Rosa: Punto y seguido (ríe).

Figura 6. Fragmento de interacción del G2 (S4).

En la S4 encontramos enunciados sobre flexión verbal, también sin reflexión metalingüística explícita. Una vez más, cuando alguien tiene una duda o detecta algún desajuste en lo que dice el compañero, realiza una reformulación simple o expresa la duda sin que tenga lugar ningún tipo de discusión. En el siguiente ejemplo (Figura 7) Marina introduce parlasen, forma incorrecta del subjuntivo parlar (en español, hablar) que puede haber construido mentalmente a imitación del castellano (hablasen) o por interferencia de la forma del estándar del catalán oriental (parlessin). La detección del error por parte de Rosa y la rectificación no genera conversación sobre la lengua.

147. Marina: Parlasen.

148. Rosa: Que no parlaren?

149. Marina: Parla? Sí. Que no parlaren català.

Figura 7. Fragmento de interacción del G2 (S4).

Podríamos pensar que la conversación no avanza porque atañe a un aspecto formal. Sin embargo, sucede lo mismo con enunciados sobre construcciones sintácticas en el fragmento de la Figura 8, al que también asignamos la etiqueta de la categoría de gramática. En el turno 147, Marina dicta el verbo de la oración a su compañera (es feia, en español se hacía). Como la planificación la realiza mentalmente Marina, es Rosa quien, mientras copia lo que dice su compañera, imagina 
la continuación de la frase y duda entre la forma impersonal y la marcada. Ante la propuesta de reformulación de Rosa, que se manifiesta con la expresión de la duda, Marina impone la solución y prosigue con la textualización sin dar pie al debate.

147. Marina: Es feia.

148. Rosa: ¿Es feia o feien?

149. Marina: Feia.

Figura 8. Fragmento de interacción del G2 (S4).

Como en las dos primeras sesiones de la secuencia el estilo de trabajo del G5 es mucho más colaborador que el del G2, el diálogo del G5 nos brinda algunos ejemplos, aunque escasos, de reflexión sobre la lengua. El desarrollo de la SD pone de manifiesto las grandes dificultades a las que se enfrenta la pareja, que responde a la tarea con evidente confusión. Aun así, cabe destacar la movilización de tímidas estrategias que les permiten superar obstáculos en la comprensión lectora y en la composición escrita. Observemos un ejemplo. El procedimiento de trabajo de Víctor y Juan Luis consiste en escribir el texto a medida que leen las fuentes de documentación. Esto desencadena discusiones entre ambos sobre la selección de la información, dado que este método no da margen a la comprensión, paso previo para una correcta planificación. En este extracto (ver Figura 9), Víctor advierte que Juan Luis ha malinterpretado un fragmento del texto, que atribuye erróneamente a un enunciador, y explica a su compañero cómo debería leerse. La explicación contiene un razonamiento de tipo metalingüístico:

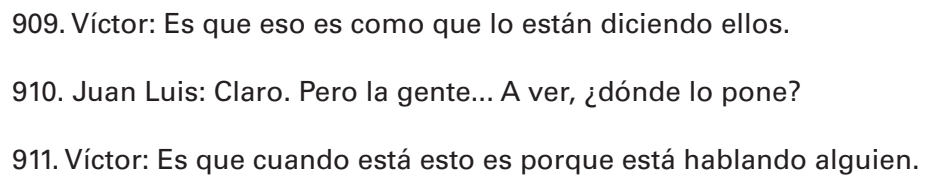

Figura 9. Fragmento de interacción del G5 (S3).

Víctor se refiere a las comillas («»), un signo ortotipográfico que identifica en la fuente que están leyendo, como el indicador de la introducción de una voz en discurso directo: «es como que lo están diciendo ellos» y «cuando está esto [las comillas] es porque está hablando alguien». De nuevo, constatamos que los alumnos no tienen el hábito de recurrir a los términos lingüísticos adecuados, incluso cuando manifiestan un conocimiento declarado sobre los fenómenos a los que aluden.

Otro episodio que nace de la dificultad encontrada en uno de los textos fuente es el de la Figura 10, esta vez en la S4. En esta sesión, uno de los elementos que más enunciados metalingüísticos desencadena es la detección de un vacío informativo en un documento. Entre los turnos 
1109 y 1114 discuten sobre cómo interpretar el contenido de la fuente con un objetivo muy claro: decidir cómo trasladar a su texto este dato sin que presente ambigüedades. Entre los turnos 1114 y 1119 la pareja debate cómo situar temporalmente unos hechos si se desconoce las fechas concretas. Aunque no adquiere un grado elevado de abstracción, el razonamiento se apoya en el uso para discernir entre las diferentes opciones que se plantean.

1109. Víctor: ¡Es que no pone la fecha, tío!

1110. Juan Luis: Sí, el 28 de marzo.

1111. Víctor: No, ¿pero de qué año? Porque aquí pone la década del 70. Y ya está, no pone nada más.

1112. Juan Luis: En la década del 70.

1113. Víctor: Sí, ¿pero qué... qué año exactamente?

1114: Juan Luis: Bueno, en la década del 70. No hay por qué decir un año concreto.

1115. Víctor: Ah, entonces todos los años, en la década del 70, el mismo 28 de marzo sufrían atentados. ¡Todos! ¡El mismo día!

1116. Juan Luis: ¡No! En la llibreria Tres i Quatre un 28 de març de la dècada dels 70 van sofrir un atemptat.

1117. Víctor: ¡Joder!

1118. Juan Luis: (ríe) ¡Es lo único que puedo arreglar! ¿Qué quieres que haga, milagros? ¡Si no lo pone!

1119. Víctor: No, no, pero es que nos dan textos incompletos, ¡tío!

Figura 10. Fragmento de interacción del G5 (S4).

La segunda secuencia de reformulaciones de la S4 se produce durante la escritura del párrafo final (Figura 11) e incluye enunciados de contenido gramatical (concretamente, de construcción sintáctica) y de contenido semántico (precisión). Las reformulaciones gramaticales son simples o con negación explícita, sin que se verbalice la reflexión metalingüística. Como se observa, entre los enunciados 1252 y la primera frase del 1256, Víctor y Juan Luis dudan entre hi va..., hi..., van ocórrer (ocurrieron) y hi va haver (hubo). Las reformulaciones semánticas son simples, con negación explícita y con una breve reflexión categórica («es que en realidad no son terroristas»), pero no van acompañadas de términos metalingüísticos explícitos (turno 1256). 


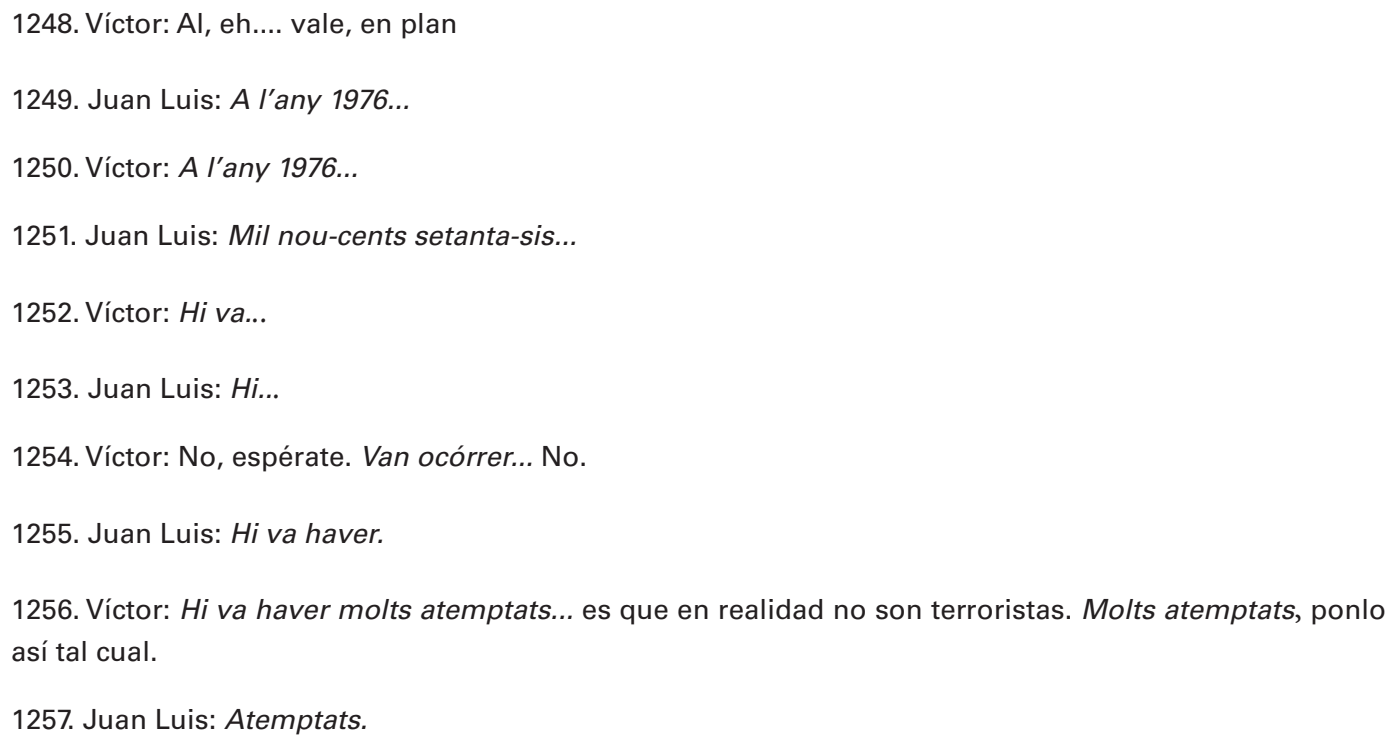

Figura 11. Fragmento de interacción del G5 (S4).

\subsection{Análisis de las interacciones de la sesión final}

En la sesión final, Marina y Rosa, del G2, cooperan en todo momento. Están decididas a presentar un producto de calidad. El cómputo de EM por categorías demuestra que prestan atención a aspectos lingüísticos, textuales y discursivos. Las cifras totales y relativas evidencian que las intervenciones de esta temática son mayoritarias durante toda la sesión. Los fragmentos siguientes dan prueba de la intensidad de la conversación entre las alumnas. Este primer ejemplo (Figura 12) se produce mientras planifican la introducción del texto final. La pareja se guía por el documento de consejos de escritura del texto final y tiene muy en cuenta las observaciones que les hicieron sus compañeros el día anterior (S5) durante la revisión entre iguales. El registro de las valoraciones del otro grupo lo conservan escrito en la tabla de revisión, que tienen sobre la mesa. 
824. Marina: A ver, quiero empezar a hablar sobre Franco.

825. Rosa: Podríamos explicar quién es primero. Porque aquí nos pusieron mal por no decir quién era. [se refiere a la tabla de revisión que rellenaron los compañeros]

826. Marina: Claro, ¿pero cómo vamos a empezar? "Franco era..."

827. Rosa: Es que aquí dice que hay que situar a los lectores en el momento histórico [se refiere a los consejos de escritura]. Entonces tienen que saber quién hizo eso, en qué año, eh... por qué lo hizo, más o menos...

828. Marina: Empezamos diciendo “a I’any no sé cuántos..." y luego ya hacemos lo que habíamos dicho del texto, pero sin poner fechas, diciendo en esta fecha, en esta fecha, en esta fecha.

$[\ldots]$

831. Rosa: ¿Y lo de los conectores?

832. Marina: ¿Se empieza diciendo en o $a$ ? Ah, conectores, pues... eh... [consulta un documento de apoyo a la escritura] ¿Per començar?

833. Rosa: No sé, es que es una introducción. Lo de per començar podríamos utilizarlo cuando vayamos a hacer ya el texto.

834. Marina: Vale, pues lo utilizamos después.

Figura 12. Fragmento de interacción del G2 (S6).

La pareja está tan inmersa en el proceso que atiende a la vez a cuestiones de niveles diversos, muy a menudo relacionadas. Fijémonos en la manera como, mientras planifican la introducción y hacen las primeras propuestas de textualización, tienen en cuenta la revisión de los compañeros (824-825), que les alerta de un vacío informativo. Al mismo tiempo, se guían por los consejos de escritura y hacen una selección del contenido pensando en las necesidades informativas de los destinatarios (826-827). Un último detalle lo encontramos en el fragmento final del ejemplo, en el que mientras planifican la organización del texto (828) deciden que utilizarán conectores (831), se plantean una duda lingüística (832), buscan el repertorio de conectores (832), y deciden en qué momento o parte del texto encajaría el conector (833-834).

A menudo se intercalan diferentes tipos de enunciados que tratan sobre un mismo fragmento del texto. La perspectiva que adoptan y la dimensión a la que se refieren determina la categoría de la intervención. En este otro ejemplo (Figura 13) se entremezclan enunciados sobre gramática (cohesión) y sobre contenido semántico (precisión). Más adelante, las aprendices sitúan el problema: la repetición léxica les produce extrañamiento. 
1006. Rosa: Va marcar una nova era. Y entre comillas el franquisme. ¡Chan, chan, chan! Ahora dejas un renglón y empiezas. El franquisme va ser una dictadura... Espérate, esto no tiene sentido, ¿eh? A ver, hemos dicho pero el golpe de estado de Franco va marcar una nueva era. El franquisme. El franquisme va ser una dictadura governada per Franco.

1007. Marina: Eh... vale, pues podríamos cambiar y en vez de el franquisme decir eh... Este... ¿movimiento? ¿Aquest moviment va ser?

1008. Rosa: ¿Era un movimiento? ¿No era una dictadura?

1009. Marina: Movimiento franquista, ¿no?

1010. Rosa: Pero entonces sería aquest moviment va ser una dictadura...

1011. Marina: Sí.

1012. Rosa: Governada per Franco, coma. ¡Es que no tiene sentido, por eso!

$[\ldots]$

1018. Marina: Si lo lees seguido. O sea, Espanya estava governada per un règim democràtic però el colp d'estat de... Francisco Franco va marcar una nova era, el franquisme. Aquest moviment va ser una dictadura governada...

1019. Rosa: Es que no nos suena bien decir eh...

1020. Marina: Otra vez Franco.

1021. Rosa: Exacto. Porque hemos dicho que el golpe de estado de Francisco Franco va marcar una nova era, el franquismo, pero es que luego se suponía que teníamos que decir que aquest moviment va ser una dictadura governada per Franco.

Figura 13. Fragmento de interacción del G2 (S6).

La S6 no es tan productiva para el G5 y los resultados que obtienen no son tan notables. Sin embargo, ahora se muestran más conscientes de la complejidad de la tarea e intentan introducir las pequeñas mejoras que están a su alcance, que afectan a niveles distintos de la lengua. En estos dos primeros ejemplos (Figuras 14 y 15) vemos cómo Víctor intenta expresar las ideas con precisión, pero no es posible acceder a la reflexión sobre la lengua bien porque no se produce o bien porque, si se produce, no se explicita. El tercer caso (Figura 16) ejemplifica un procedimiento de imitación los textos académicos. Mientras textualiza, Víctor recurre a la deíxis textual formada por el participio nombrados seguido del adverbio anteriormente (esmentats anteriorment, en catalán). Él mismo afirma que «eso siempre queda bien».

1714. Víctor: ¿Se podría decir una dictadura governada per Franco? (19 segundos)

1715. Juan Luis: ¿Cómo se llamaba de nombre normal? ¿Franco qué más?

Figura 14. Fragmento de interacción del G5 (S6). 
1915. Víctor: Que la dictadura supuso... No, ¡supuso no! ¡Hizo! Hizo un gran mal a la... a la lengua del catalán, y... que... per culpa de... que per culpa de l'extrema dreta moltes persones... que només volien...

Figura 15. Fragmento de interacción del G5 (S6).

1844. Víctor: L'extrema dreta pel seu compte va organitzar els atemptats...

1845. Juan Luis: ¿Va organitzar?

1846. Víctor: Va organitzar els atemptats esmentats anteriorment. Que eso siempre queda bien.

Figura 16. Fragmento de interacción del G5 (S6).

Los enunciados más numerosos son los gramaticales y surgen durante la textualización, pero no se producen reflexiones explícitas, dado que los problemas se resuelven de manera expeditiva, mediante reformulaciones simples. A veces, la movilización de contenidos gramaticales (los conectores) les sirve como estrategia de apoyo para organizar el discurso (superestructura) (ver Figura 17):

1627. Víctor: Eh... primer de-A ver, yo pondría primer de tot al 1936...

1628. Juan Luis: Sí, la repressió va suponer para la llengua una represión...

1629. Víctor: Sí. Pero primer de tot o... eh... la repressió de la llengua va començar, o algo así.

1630. Juan Luis: ¿La qué?

1632. Víctor: Que teníamos que poner en plan primero, eh..., en plan... comenzó en tal... luego segueix con la...

Figura 17. Fragmento de interacción del G5 (S6).

Uno de los pocos ejemplos de reflexión metalingüística con terminología específica de esta sesión está motivado por las regulaciones externas (Figura 18). En la sesión de revisión entre iguales el equipo evaluador les cuestiona la organización del texto, que consideran caótica. En la S6, la investigadora les entrega los consejos para la escritura de la versión final, que también apelan al orden lógico de los hechos. La alusión al salto temporal, cosecha propia de la pareja del G5, revela una percepción evidente del problema que implica la propuesta de Juan Luis. Aun así, la discusión no se desarrolla.

1763. Juan Luis: Mira, podemos poner esto. Eh... tal, no sé cuántos. ¿Pongo eso? Espera, vamos a poner esto.

1764. Víctor: ¿Seguro? ¿Quieres hacer un salto temporal tan grande?

Figura 18. Fragmento de interacción del G5 (S6). 


\section{Conclusiones}

Del presente análisis se desprenden las siguientes conclusiones, que ordenamos de acuerdo con los tres objetivos:

1. Detectar en qué momentos de la secuencia didáctica se produce la actividad metalingüística. El análisis de los enunciados metalingüísticos durante la escritura demuestra que la actividad metalingüística se produce a lo largo de las tres sesiones analizadas. La conversación entre los escritores durante el trabajo en colaboración permite conocer con detalle cómo se desarrolla el proceso de aprendizaje.

2. Conocer el contenido de esta actividad y su evolución a lo largo de la SD. En términos globales, los tipos de EM que predominan son la superestructura, la gramática, el contenido semántico y los aspectos gráficos y sonoros. La tarea de escribir a partir de fuentes exige que hablen sobre el contenido, su expresión y la construcción del texto propio. A la hora de textualizar, y cuando revisan, se enfrentan a obstáculos gramaticales y se plantean dudas ortográficas. El análisis de los ejemplos de interacción expuestos muestra que, a pesar de que hay numerosas manifestaciones de actividad metalingüística en los comentarios y en las reformulaciones orales, la reflexión tiende a la superficialidad. La reflexión metalingüística explícita no se produce con frecuencia, y cuando se halla suele verbalizarse mediante lenguaje de uso común. Los alumnos no están acostumbrados a hablar sobre la lengua y la SD no incluye actividades de sistematización de contenidos que les ayuden a hacerlo. No obstante, tras las dinámicas de regulación externa se observa un aumento del grado de conciencia sobre el texto. Es evidente que la observación guiada, aunque únicamente se haya vinculado al uso (la mejora de la producción escrita), desencadena actividad metalingüística.

3. Identificar los factores que fomentan la actividad metalingüística. Encontramos, en primer lugar, la cooperación. El estilo de trabajo del G5 cambia poco en las tres sesiones, hecho que explica que no haya grandes fluctuaciones de EM. Por el contrario, el G2 adopta tres modalidades de trabajo diferentes para cada una de las tres sesiones, de menor a mayor colaboración, lo cual se ve reflejado en el número de EM registrados cada día. Igualmente, la implicación en la tarea fomenta una conversación más rica. El interés de los estudiantes se manifiesta en su afán por resolver los diferentes problemas que plantea la escritura del texto, especialmente el final. Esta cuestión conecta con un tercer factor importante: las regulaciones externas del final de la secuencia, introducidas en forma de dinámicas de revisión y de reescritura que se apoyan en los instrumentos de la ficha de revisión y los consejos de escritura del texto definitivo. La aparición de estos elementos promueve un trabajo más focalizado que se traduce en episodios de gran dinamismo y recursividad en las operaciones de planificación, textualización y revisión. Los aprendices empiezan a concebir el texto en su complejidad, se plantean cuestiones sobre niveles diversos del texto (notablemente sobre aspectos que antes no habían considerado) y hablan sobre ellos. Por último, destaca el papel de la tarea híbrida y situada, porque su complejidad - acoplar el contexto retórico y el contenido (Bereiter y Scardamalia, 1987) - exige pensar y hablar sobre lo que se escribe y reformular lo escrito, y puede derivar en un uso más consciente. Manejar conceptos propios de disciplinas académicas y referirse a ellos exige una reflexión profunda sobre el contenido y sobre las formas lingüísticas que permiten expresar las ideas que se generan. 


\section{Referencias bibliográficas}

Allal, L. (2000). Regulación metacognitiva de la escritura en el aula. En M. Milian y A. Camps (Eds.), El papel de la actividad metalingüistica en el aprendizaje de la escritura (pp. 187-214). Buenos Aires: Homo Sapiens Ediciones.

Bereiter, C., y Scardamalia, M. (1987). The psychology of written composition. Hillsdale, NJ: Erlbaum.

Camps, A. (Coord.) (2003). Secuencias didácticas para aprender a escribir. Barcelona: Graó.

Camps, A. (2017). Reflexiones sobre la enseñanza y el aprendizaje de la gramática. En A. Camps y T. Ribas (Coords.), El verbo y su enseñanza. Hacia un modelo de enseñanza de la gramática basado en la actividad reflexiva (pp. 19-31). Barcelona: Octaedro.

Camps, A. (2020). Proyectos de lengua entre la teoría y la práctica. En A. Camps y X. Fontich (Eds.), La actividad metalingüistica como espacio de encuentro de la escritura y la gramática: Un itinerario de enseñanza e investigación en educación lingüística (pp. 63-71). San Juan: Universidad Nacional de San Juan.

Camps, A., y Milian, M. (2020). La actividad metalingüística en el aprendizaje de la composición escrita. En A. Camps y X. Fontich (Eds.), La actividad metalingüistica como espacio de encuentro de la escritura y la gramática: Un itinerario de enseñanza e investigación en educación lingüística (pp. 115-125). San Juan: Universidad Nacional de San Juan.

Camps, A., Guasch, O., Milian, M., y Ribas, T. (2020). Actividad metalingüística durante el proceso de producción de un texto argumentativo. En A. Camps y X. Fontich (Eds.), La actividad metalingüística como espacio de encuentro de la escritura y la gramática: Un itinerario de enseñanza e investigación en educación lingüística (pp. 133-143). San Juan: Universidad Nacional de San Juan.

Carlino, P. (2013). Alfabetización académica diez años después. Revista Mexicana de Investigación Educativa, 18(57), 355-381.

Culioli, A. (1990). Pour une linguistique de l'éntonation (vol. 1). París: Ophrys.

Flower, L. (1990). The role of task-representation in reading-to-write. En L. Flower, V. Stein, J. Ackerman, M.J. Kantz, K. McCormick y W.C. Peck (Eds.), Reading-to-write: Exploring a cognitive and social process (pp. 35-75). Nueva York: Oxford University Press.

Fontich, X. (2016). L1 Grammar Instruction and Writing: Metalinguistic Activity as a Teaching and Research Focus. Language and Linguistics Compass, 10/5, 238-254. https://doi.org/10.1111/ $\underline{\operatorname{lnc} 3.12184}$

Fontich, X., y Camps, A. (2014). Towards a rationale for research into grammar teaching in schools. Research papers in education, 29(5), 598-625. https://doi.org/10.1080/02671522.2 $\underline{013.813579}$

Fontich, X. y García Folgado, M. J. (2018). Grammar instruction in the Hispanic area: The case of Spain with attention to empirical studies on metalinguistic activity. L1-Educational Studies in Language and Literature, 18, 1-39. DOI: 10.17239/L1ESLL-2018.18.04.02

Gombert, J. É. (1990). Le développement métalinguistique. París: PUF. 
Graham, S., Liu, K., Bartlett, B., Ng, C., Harris, K. R., Aitken, A., Barkel, A., Kavanaugh, C., y Talukdar, J. (2018). Reading for writing: A meta-analysis of the impact of reading and reading instruction on writing. Review of Educational Research, 88, 243-284. https://doi. org/10.3102/0034654317746927

Karmiloff-Smith, C. (1992). Beyond modularity. A developmental perspective on cognitive science. Cambridge: MIT Press.

Mercer N., y Littleton, K. (2007). Dialogue and the development of children's thinking. a sociocultural approach. Nueva York: Routledge.

Myhill, D. (2011). 'The Ordeal of Deliberate Choice': Metalinguistic Development in Secondary Writers. En V. Berninger (Ed.), Past, present, and future contributions of cognitive writing research to cognitive psychology (pp. 247-274). Nueva York: Psychology Press/Taylor Francis Group.

Myhill, D., y Jones, S. (2015). Conceptualizing metalinguistic understanding in writing. Cultura y Educación, 27(4), 839-867. https://doi.org/10.1080/11356405.2015.1089387

Ribas, T. (2000). Lavaluació formativa de la composició escrita en l'ensenyament i l'aprenentatge de la composició escrita. Anàlisi de la incidència de la pauta d’avaluació en el procés de composició escrita (Tesis doctoral, Universitat Autònoma de Barcelona). Recuperado de: https://www. tdx.cat/handle/10803/4680

Ribas, T. (2020). La regulación del proceso de composición escrita en grupo: análisis de la utilización de las pautas de revisión. En A. Camps y X. Fontich (Eds.), La actividad metalingüística como espacio de encuentro de la escritura y la gramática: Un itinerario de enseñanza e investigación en educación lingüística (pp. 51-61). San Juan: Universidad de San Juan.

Rodríguez Gonzalo, C. (2012). La enseñanza de la gramática: las relaciones entre la reflexión y el uso lingüístico. Revista Iberoamericana de Educación, 59(1), 87-118. https://doi.org/10.35362/ $\underline{\text { rie590458 }}$

Zayas, F. (2012a). Los géneros discursivos y la enseñanza de la composición escrita. Revista Iberoamericana de Educación, 59, 63-85. https://doi.org/10.35362/rie590457

Zayas, F. (2012b). 10 ideas clave. La competencia lectora según PISA. Reflexiones y orientaciones didácticas. Barcelona: Graó. 